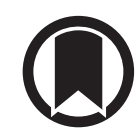

CrossMark

\title{
Proliferating SPP1/MERTK-expressing macrophages in idiopathic pulmonary fibrosis
}

\author{
Christina Morse ${ }^{1,7}$, Tracy Tabib ${ }^{1,7}$, John Sembrat ${ }^{2}$, Kristina L. Buschur ${ }^{3}$, \\ Humberto Trejo Bittar ${ }^{4}$, Eleanor Valenzi ${ }^{2}$, Yale Jiang ${ }^{5,6}$, Daniel J. Kass (1) ${ }^{2}$ \\ Kevin Gibson ${ }^{2}$, Wei Chen ${ }^{5}$, Ana Mora ${ }^{2}$, Panayiotis V. Benos ${ }^{3}$, Mauricio Rojas ${ }^{2,8}$ \\ and Robert Lafyatis (1) ${ }^{1,8}$
}

Affiliations: 'Division of Rheumatology and Clinical Immunology, University of Pittsburgh, Pittsburgh, PA, USA. ${ }^{2}$ Division of Pulmonary, Allergy and Critical Care, Dept of Medicine, University of Pittsburgh, Pittsburgh, PA, USA. ${ }^{3}$ Dept of Computational and Systems Biology, University of Pittsburgh, Pittsburgh, PA, USA. ${ }^{4}$ Dept of Pathology, University of Pittsburgh, Pittsburgh, PA, USA. ${ }^{5}$ Division of Pulmonary Medicine, Allergy and Immunology, Dept of Pediatrics, School of Medicine, University of Pittsburgh, Pittsburgh, PA, USA. 'School of Medicine, Tsinghua University, Beijing, China. ${ }^{7}$ Authors contributed equally to this work. ${ }^{8}$ Authors contributed equally to this work.

Correspondence: Robert Lafyatis, 200 Lothrop St, BST S720, Pittsburgh, PA 15260, USA. E-mail: lafyatisđapitt.edu

@ERSpublications

By single-cell RNA-sequencing we identify three discrete pulmonary macrophage subsets, including one expressing highly upregulated SPP1 and proliferating in fibrotic IPF lower lobes, accompanied by marked deposition of osteopontin in the matrix http://bit.ly/2wIRNqF

Cite this article as: Morse C, Tabib T, Sembrat J, et al. Proliferating SPP1/MERTK-expressing macrophages in idiopathic pulmonary fibrosis. Eur Respir J 2019; 54: 1802441 [https://doi.org/10.1183/ 13993003.02441-2018].

ABSTRACT A comprehensive understanding of the changes in gene expression in cell types involved in idiopathic pulmonary fibrosis (IPF) will shed light on the mechanisms underlying the loss of alveolar epithelial cells and development of honeycomb cysts and fibroblastic foci. We sought to understand changes in IPF lung cell transcriptomes and gain insight into innate immune aspects of pathogenesis.

We investigated IPF pathogenesis using single-cell RNA-sequencing of fresh lung explants, comparing human IPF fibrotic lower lobes reflecting late disease, upper lobes reflecting early disease and normal lungs.

IPF lower lobes showed increased fibroblasts, and basal, ciliated, goblet and club cells, but decreased alveolar epithelial cells, and marked alterations in inflammatory cells. We found three discrete macrophage subpopulations in normal and fibrotic lungs, one expressing monocyte markers, one highly expressing FABP4 and INHBA (FABP $\left.4^{\mathrm{hi}}\right)$, and one highly expressing SPP1 and MERTK (SPP1 $\left.1^{\mathrm{hi}}\right)$. SPP1 $1^{\mathrm{hi}}$ macrophages in fibrotic lower lobes showed highly upregulated SPP1 and MERTK expression. Low-level local proliferation of SPP1 ${ }^{\text {hi }}$ macrophages in normal lungs was strikingly increased in IPF lungs.

Co-localisation and causal modelling supported the role for these highly proliferative SPP1 ${ }^{\text {hi }}$ macrophages in activation of IPF myofibroblasts in lung fibrosis. These data suggest that SPP $1^{\text {hi }}$ macrophages contribute importantly to lung fibrosis in IPF, and that therapeutic strategies targeting MERTK and macrophage proliferation may show promise for treatment of this disease.

This article has supplementary material available from erj.ersjournals.com

Received: 03 Jan 2019 | Accepted after revision: 18 May 2019

Copyright OERS 2019 


\section{Introduction}

The complex pathogenesis of idiopathic pulmonary fibrosis (IPF) involves multiple cell types [1], namely the injury and loss of alveolar epithelial cells [2, 3]; formation of honeycomb cysts lined by several epithelial cell lineages ( $163^{+}$, goblet and ciliated cells) [4]; expansion of reparative basal stem cells [5-7]; and development of fibroblastic foci composed of myofibroblasts acting as fibrotic effector cells [8].

In murine bleomycin-induced pulmonary fibrosis, several studies suggest that inflammation and monocyte-derived macrophages drive fibrosis, through overactive reparative responses to alveolar cell injury. CCR2 deletion depletes bleomycin-induced pulmonary macrophages and fibrosis [9]. Similarly, deletion of CD11b-expressing monocyte-derived macrophages ameliorates bleomycin-induced lung fibrosis [10]. A recent study has indicated that monocyte-derived alveolar macrophages drive bleomycin-induced lung fibrosis [11].

Recent studies show that in most tissues resident macrophages are derived from embryonic progenitors. Alveolar macrophages develop from embryonic progenitors originating in the yolk sac and fetal liver [12, 13]. Interstitial macrophages also derive from yolk sac and fetal liver macrophages, although a second group of interstitial monocyte/macrophages appears to derive from circulating monocytes [13, 14]. Thus, in normal adult mouse lungs most resident macrophages are derived from embryonic progenitors, but a subset of interstitial cells derives from monocytes. Multiple studies have shown that murine macrophages are capable of self-renewal. Tissue-resident macrophages have a long life span and proliferate under the influence of macrophage colony-stimulating factor (M-CSF) and granulocyte-macrophage colony-stimulating factor (GM-CSF) [15, 16], and interleukin (IL)-4 stimulates resident macrophage proliferation during nematode infections [17]. M-CSF is required for proliferation of both inflammatory bone marrow-derived macrophages and resident macrophage populations in zymosan-induced peritonitis [18]. Whether proliferation of resident macrophages contributes to human IPF is less studied.

We used single-cell RNA-sequencing (scRNA-seq) to provide a comprehensive view of altered cell numbers and transcriptomes associated with IPF. As a criterion for IPF disease progression we examined changes in upper (early disease) and lower (late fibrotic disease) lobes compared to normal lungs. We identify subpopulations of macrophages in normal lungs that in IPF show increased proliferation and highly upregulated expression of SPP1, suggesting that these cells play an important role in IPF pathogenesis.

\section{Methods}

Healthy control lungs were obtained under a protocol approved by the University of Pittsburgh Committee for Oversight of Research and Clinical Training Involving Decedents, following rejection as candidate donors for transplant. IPF lung tissue was obtained under a protocol approved by the University of Pittsburgh institutional review board, during transplantation surgery. Detailed methods are included in the supplementary material. scRNA-seq data can be accessed at the Gene Expression Omnibus: GSE128033.

\section{Results}

\section{Macrophage subpopulations in normal lungs}

We analysed single-cell transcriptomes of 24220 cells from seven normal lung samples using scRNA-seq, including one technical replicate together with $\mathrm{t}$-distributed stochastic neighbour embedding (t-SNE) and coloured by groups of cells (supplementary table S1). This analysis showed 16 different clusters of cells (figure 1a), using previously described markers, including inflammatory, epithelial, vascular and mesenchymal cell types, as indicated (supplementary figures S1 and S2, table S2 and supplementary results). Cells identified by the subject of origin ensured that clusters included cells from each sample (figure 1b).

Normal control lungs showed three discrete populations of monocyte/macrophages, all of which expressed high levels of CD163 and AIF1 (supplementary figure S1 and figure 1a, clusters 0,1 and 3). The first group of macrophages (FABP $4{ }^{\text {hi }}$ macrophages, figure 1a-d, cluster 0 ) expressed high levels of FABP4 and INHBA, and relatively low levels of SPP1, MERTK, LGMN and SIGLEC10. A second group of macrophages (SPP1 ${ }^{\text {hi }}$, figure $1 \mathrm{a}-\mathrm{d}$, cluster 1 ) expressed relatively high levels of SPP1, as well as MERTK, LGMN and SIGLEC10 and relatively low levels of FABP4 and INHBA. A third macrophage population $\left(F C N 1^{h i}\right.$ monocyte/macrophages) expressed relatively high levels of $F C N 1$, as well as several marker genes associated with monocytes: CD14, IL1B, INSIG1, OSM, IL1R2 and THBS1 [14], and low to no expression of FABP4, INHBA, MERTK and SPP1 (figure 1a-d, cluster 3, and data not shown). Previously defined flow cytometry markers did not distinguish these subsets well (supplementary figure S3). A fourth population of cells adjacent to the macrophage populations discretely expressed CD1C and therefore most 

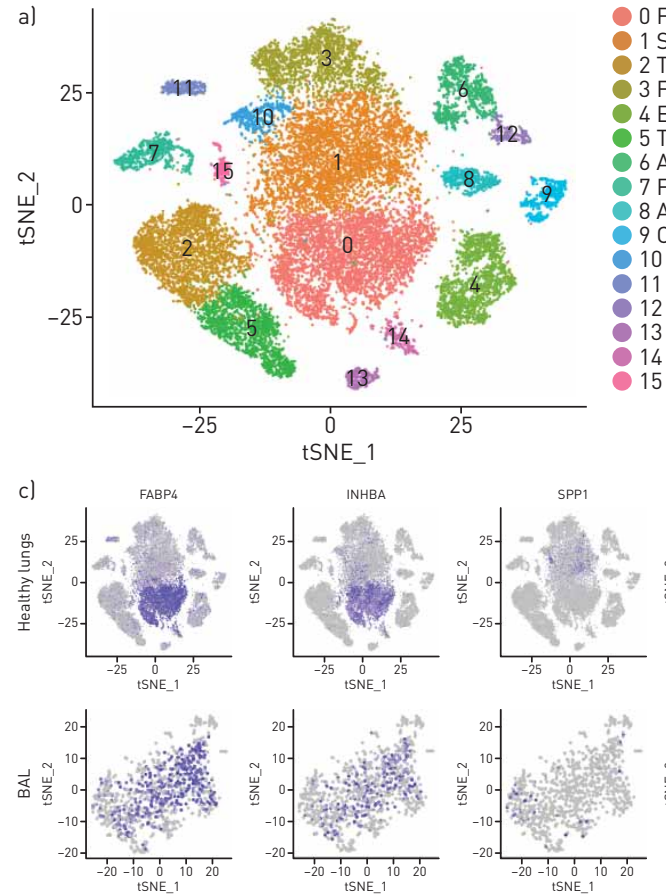

Healthy lung-cluster numbers

d)
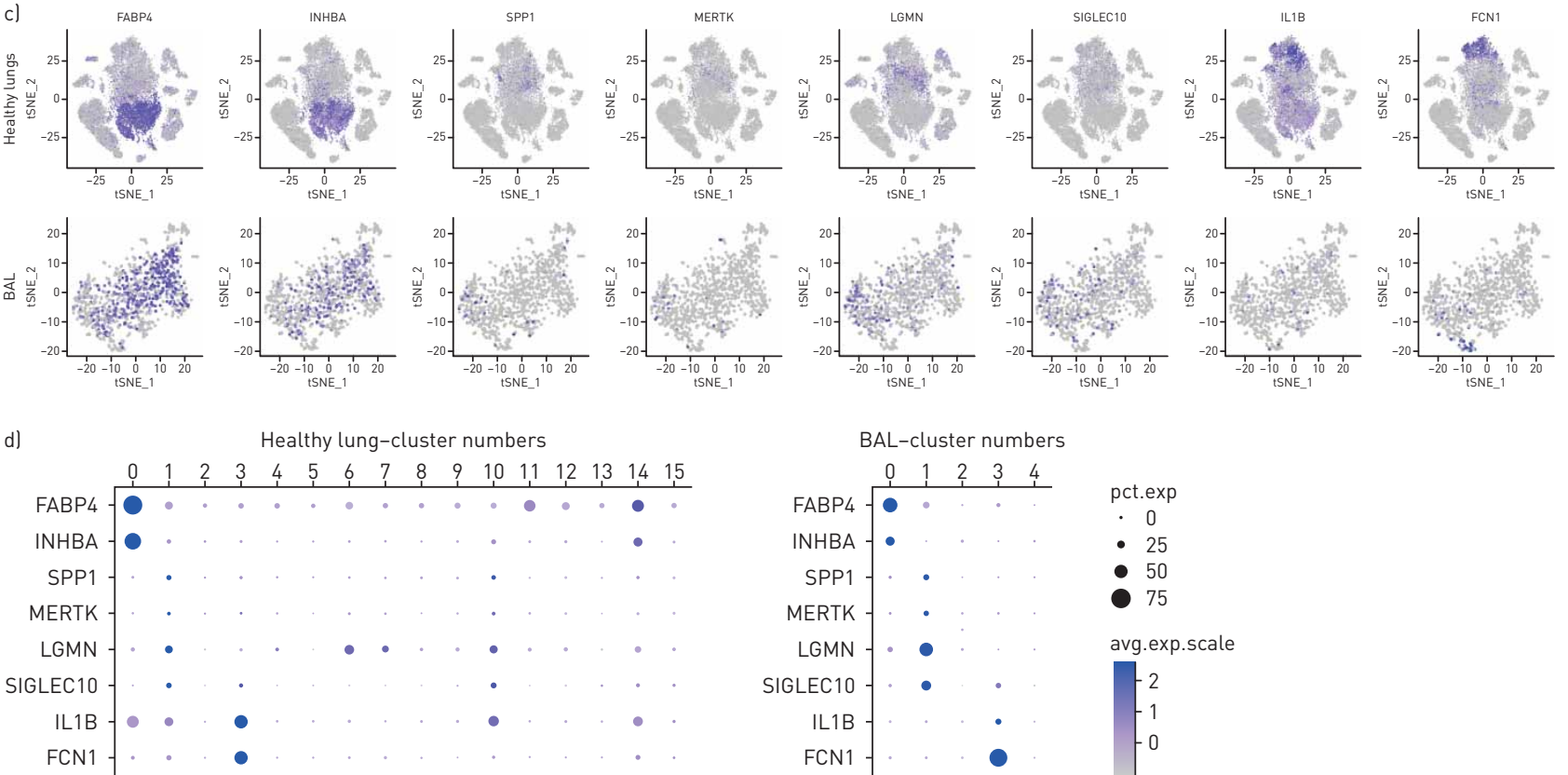

BAL-cluster numbers
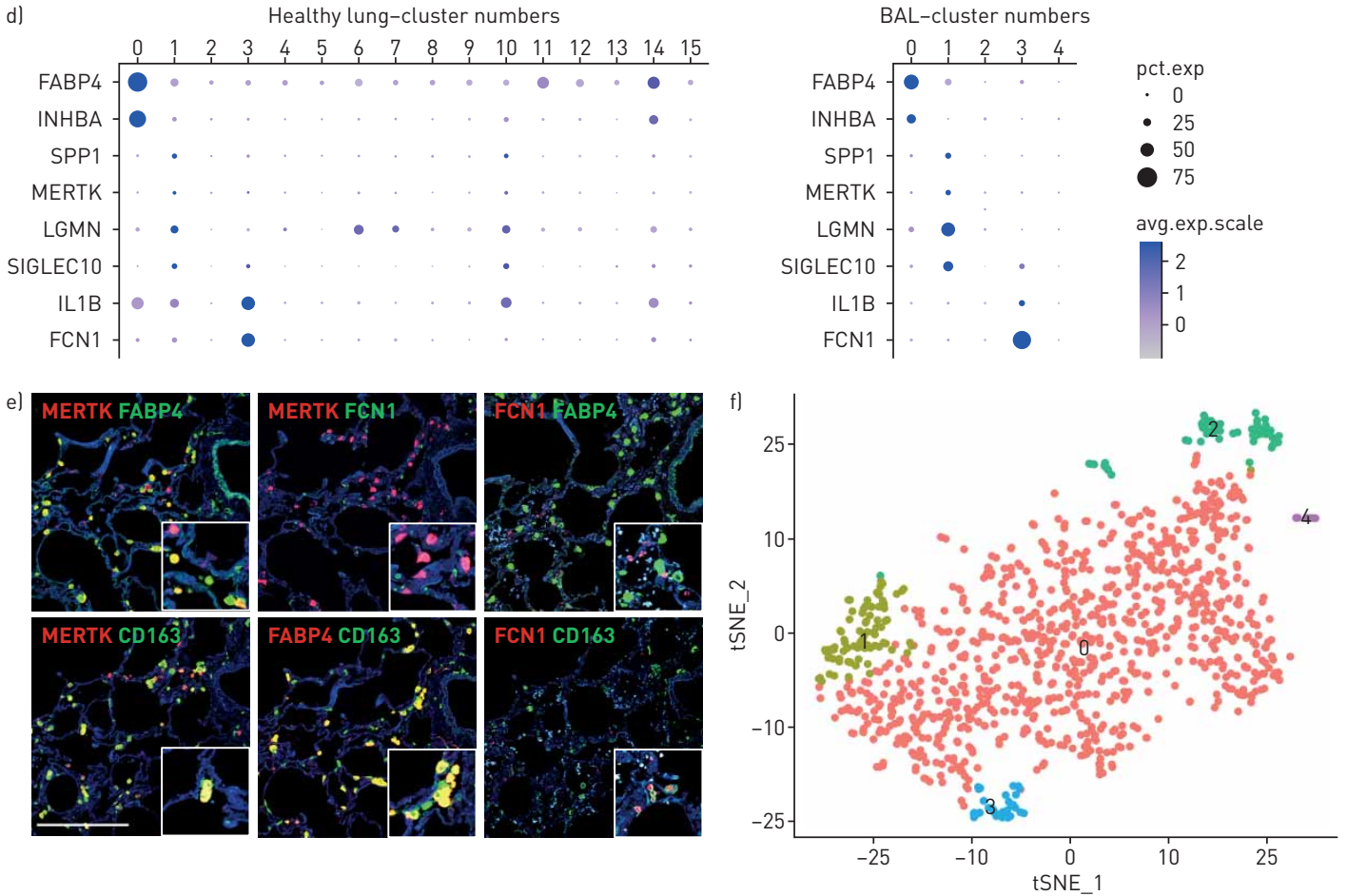

FIGURE 1 Characterisation of normal lung macrophages. T-distributed stochastic neighbour embedding (T-SNE) of normal lung cells indicates 10

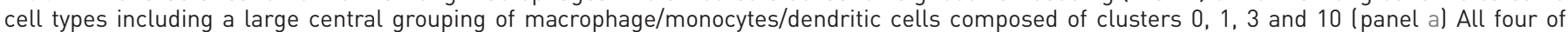
these clusters express AIF1 and all but the dendritic cell cluster (cluster 10) express CD163 (supplementary figure S1). The subject of origin for each of the samples in indicated by a different colour in panel b. The three different macrophage/monocyte clusters (clusters 0 , 1 and 3 ) can be distinguished in feature plots (panel c, purple intensity indicates level of gene expression) and dot plots (panel $d$, dot size indicates percent of

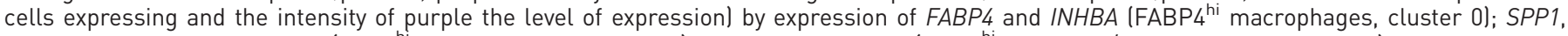
MERTK, LGMN and SIGLEC10 (SPP1 ${ }^{\text {hi }}$ macrophages, cluster 1); or ILIB and FCN1 (FCN1 ${ }^{\text {hi }}$ monocyte/macrophages, cluster 3). Dendritic cells, expressing $C D 1 C$, are found in cluster 10 (see also supplementary figure S1). Macrophage monocyte cell types in bronchoalveolar lavage (BAL)

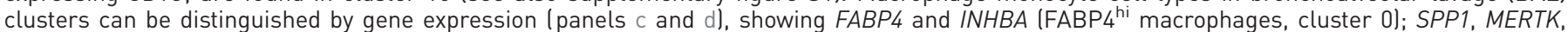

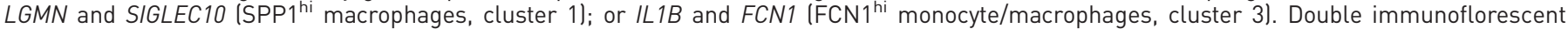

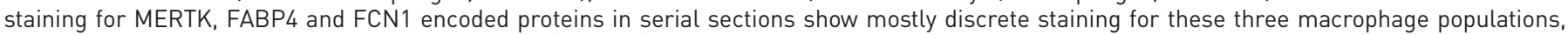
all three co-stain for CD163. Scale bar=500 $\mu \mathrm{m}$ (panel e). BAL cells from a healthy subject clustered by t-SNE show discrete clusters of macrophages (panel f). 
likely represented dendritic cells (figure 1a, supplementary figures S1 and S2; cluster 10). Re-clustering only the myeloid cells showed the same cell subsets (supplementary figures S4 and S5).

Immunofluorescent staining of normal lungs showed that discrete cells co-stained for CD163 and either FABP4 or SPP1/osteopontin (not shown). Although SPP1-expressing cells were detected, staining of SPP1/ osteopontin, a matricellular protein, also stained extracellular matrix. Immunofluorescent staining of MERTK better identified the SPP $1^{\text {hi }}$ subset of macrophages that was generally discrete from FABP4 staining of $\mathrm{FABP} 4^{\mathrm{hi}}$ macrophages, although some overlap in protein expression of each of these markers was seen, as was seen at the level of MERTK and FABP4 gene expression (figure 1e and supplementary figure S6). The staining for these markers was more discrete in IPF tissues, as described later. FCN1 staining showed smaller cells, representing $\mathrm{FCN1}^{\text {hi }}$ macrophages. All three macrophage populations could be found in the interstitial space in normal lung samples.

\section{Normal alveolar macrophages are composed primarily of FABP4 ${ }^{\text {hi }}$ macrophages}

Since morphological evaluation of immunofluorescent staining of macrophages in normal lung tissues did not provide a clear localisation of macrophage subsets between alveolar and interstitial spaces, we analysed cells from normal lung bronchoalveolar lavage (BAL) (figure 1f). The cell types identified by marker genes were all inflammatory cells, mostly macrophages, expressing CD163 and AIF1, but also discrete clusters that included both T-cells (expressing CD3D) and natural killer (NK) cells (expressing KLRF1, GNLY and NKG7, cluster 2) and B-cells (expressing MS4A1/CD20, cluster 4; supplementary figures S7 and S8). As seen in normal lung tissue, three macrophage populations could be distinguished. Most of the macrophages showed gene expression features of FABP4 ${ }^{\text {hi }}$ macrophages, expressing relatively high levels of FABP4 and INHBA, and lower levels of SPP1, MERTK, SIGLEC10, LGMN, IL1B and FCN1 (figure 1c and $\mathrm{d}$, cluster 0 ). However, minor populations of $\mathrm{SPP} 1^{\text {hi }}$ and $\mathrm{FCN} 1^{\text {hi }}$ macrophages were also seen in BAL (figure $1 \mathrm{c}, \mathrm{d}$ and $\mathrm{f}$, clusters 1 and 3 ). Clustering of the macrophages from this healthy BAL with macrophages from a second normal BAL with brushing showed essentially the same results with a predominance of FABP $4{ }^{\text {hi }}$ macrophages (supplementary figures S9 and S10).

Consistent with these observations, when the BAL cells were analysed with the normal lungs cells by $\mathrm{t}$-SNE, most of the cells clustered with the FABP $4{ }^{\text {hi }}$ macrophages (supplementary figure S11). However, on this combined analysis, consistent with t-SNE clustering of the BAL cells alone, some of the BAL macrophages clustered with the SPP $1^{\text {hi }}$ and others with the $\mathrm{FCN} 1^{\mathrm{hi}}$ macrophages (supplementary figures S10 and S11). Thus, alveolar macrophages were composed primarily of FABP4 $4^{\text {hi }}$ macrophages, but all three macrophage populations, as well as lymphocyte subsets, were found in BAL.

\section{Lung samples from patients with IPF show alterations in cellular composition compared to healthy controls}

In order to better understand the changes in populations and gene expression of cell types associated with the development of IPF, we analysed freshly digested lung explants from three patients with IPF, and three healthy controls showing the least changes on pathology and analysed using the same chemistry (V2 10X Genomics) by scRNA-seq (table 1 and supplementary table S1). Since fibrotic changes in IPF lungs typically are first evident at the lung bases and the disease then progresses apically, we analysed samples from both the right upper and lower lobes to capture disease at different points in evolution. Lower lobe pathology showed classical changes associated with IPF, including honeycomb cysts and fibroblastic foci (figure 2a), as well as smooth muscle actin staining myofibroblasts and collagen deposition (supplementary figures S12A and S12B). Two of the three IPF upper lobes showed markedly less fibrosis, and similar or more inflammation.

\section{Different cell types in normal and IPF lungs revealed by sCRNA-seq}

Single-cell transcriptomes of 47771 cells, representing 17231 cells from healthy lungs and 30540 cells from IPF lungs from the different lung samples were analysed together by t-SNE and coloured by groups of cells (figure 2b), showing 23 different cell clusters. In addition, cells were identified by the subject of origin to ensure that clusters represented cell types found in all samples (supplementary figure S13). Furthermore, cells were identified by the disease status (normal, upper lobes and lower lobes) to show the change in patterns of cell types with disease (figure 2c). Using previously described markers most clusters were identified as discrete cell types, including inflammatory, epithelial, vascular and mesenchymal cell types (supplementary results and figures S14-S16; supplementary table S3). Epithelial cell types (cluster 7, $8,9,12,14,20,21$ ) were reanalysed and low-quality cells filtered, allowing for discrimination of each epithelial cell type (figure $2 \mathrm{~d}$ and e, supplementary figure S17).

Macrophages and dendritic cells identified by AIF1 and CD163 expression were found in five different clusters (figure 2b, supplementary figures S15 and S16; clusters $0,1,6,15$ and 16). Cells in cluster 0 
TABLE 1 Lung sample analysed by single-cell RNA sequencing, demographics and associated review of pathology

\begin{tabular}{|c|c|c|c|c|c|c|}
\hline Sample ID & $\begin{array}{l}\text { Single } \\
\text { cell ID }\end{array}$ & Tissue type & Sex & $\begin{array}{l}\text { Age } \\
\text { years }\end{array}$ & $\begin{array}{l}\text { Cells } \\
\text { analysed } n\end{array}$ & Pathological assessment of adjacent lung tissue \\
\hline 2017-025-NOR & SC56 & Control lung & Female & 57 & 4334 & Normal healthy lung \\
\hline \multirow[t]{2}{*}{ 2017-064-IPF } & SC87 & $\begin{array}{l}\text { IPF lung } \\
\text { lower lobe }\end{array}$ & Female & 70 & 10479 & $\begin{array}{l}\text { UIP: acute exacerbation of UIP, diffuse alveolar damage and } \\
\text { inflammation }\end{array}$ \\
\hline & SC87 & $\begin{array}{l}\text { IPF lung } \\
\text { upper lobe }\end{array}$ & & & 4717 & $\begin{array}{l}\text { Mostly UIP; more diffuse than lower lobe. Some mixed fibrotic } \\
\text { and cellular NSIP (acute). Some honeycomb cysts }\end{array}$ \\
\hline 2017-067-IPF & $\mathrm{SC} 93$ & $\begin{array}{l}\text { IPF lung } \\
\text { lower lobe }\end{array}$ & Male & 69 & 5920 & $\begin{array}{l}\text { Classic end-stage lung: honeycomb cysts, bronchial } \\
\text { metaplastic cells, some smooth muscle metaplasia }\end{array}$ \\
\hline 2017-100-IPF & SC154 & $\begin{array}{l}\text { IPF lung } \\
\text { upper lobe }\end{array}$ & & & 4019 & $\begin{array}{l}\text { Nearly complete replacement of the lung architecture by scar } \\
\text { tissue with honeycomb change with end-stage lung disease }\end{array}$ \\
\hline
\end{tabular}

IPF: idiopathic pulmonary fibrosis; UIP: usual interstitial pneumonia; NSIP: nonspecific interstitial pneumonia; COPD: chronic obstructive pulmonary disease.

expressed FABP4 most highly and represented the same FABP4 $4^{\text {hi }}$ macrophage population described earlier in normal lungs. Cells in cluster 1 expressed SPP1 most highly and SPP $1^{\text {hi }}$ healthy control macrophages fell into this cluster, which also expressed the highest levels of MERTK, LGMN and SIGLEC10. Cells in cluster 6 expressed FCN1 most highly as well as CD14, IL1B, INSIG1, OSM, IL1R2 and THBS1, the other markers shown above to be associated with the $\mathrm{FCN1}^{\mathrm{hi}}$ monocyte/macrophage subset in normal lungs. Cluster 15 represented proliferating macrophages analysed further later. Cluster 16 represented CD1C-expressing dendritic cells, again as seen in normal lungs. Thus, this combined analysis recapitulated the macrophage/monocyte/dendritic cell subsets seen in healthy lungs.

\section{Increased and decreased proportions of cell types changed in a graded fashion between normal, upper lobe IPF and lower lobe IPF}

The proportions of different cell populations changed in IPF compared to normal lungs. In almost all cases, changes in lower lobes were more dramatic than changes in upper lobes, compared to normal lungs (figure $3 \mathrm{a}$ and $\mathrm{b}$ ). Several inflammatory cell populations decreased in this graded fashion, including FABP4 ${ }^{\text {hi }}$ and $\mathrm{FCN}_{1}{ }^{\text {hi }}$ macrophages, and NK cells. In contrast, SPP1 $1^{\text {hi }}$ macrophages trended toward modestly increased proportion in IPF lower lobes. The proportion of T-cells, B-cells and plasma cells showed little difference between normal, IPF upper and IPF lower lobes.

MUC5 $^{+}-\left(\right.$MUC5AC and MUC5B), FoxJ1 $1^{+}$(FOXJ1) and $\mathrm{p} 63^{+}-$(TP63) expressing cells, which make up honeycomb cysts [19] associated with goblet cells (cluster 9), ciliated cell (cluster 8) and basal cells (cluster 14), respectively, as well as club cells, were all highly increased in IPF lungs (figure $3 \mathrm{~b}$ ). Consistent with their marked expansion, we detected increased numbers of cells with club and basal cell markers that co-expressed markers of cell proliferation (supplementary table S4). AGER-expressing AT1 and SFTPC-expressing AT2 cells declined in fibrotic lower lobes although not reaching statistical significance (figure 3b), and we did not detect proliferating AT2 cells, although this might represent the relative paucity of AT2 cells surviving digestion [20]. We did see rare bipolar cells described to have makers of both AT1 and AT2 cells in normal or IPF lungs (supplementary figure S18); however, there were no distinctive markers for these cells and thus we are uncertain whether these represent cellular transitions or doublet cells.

The proportion of alveolar and bronchiolar epithelial cells in normal lungs appeared lower than anticipated. Overall gene expression between bulk tissue RNA-seq, RNA-seq of digested cells and combined scRNA-seq gene expression correlated well between these three groups (data not shown). However, expression of selected marker genes of cells after digestion indicated a selective loss of 
a) i)
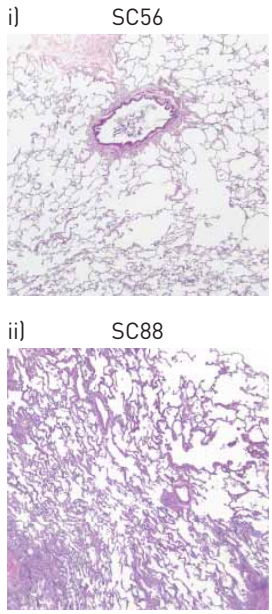

iii)

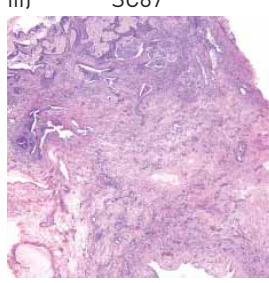

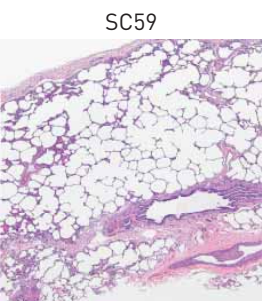

$\mathrm{SC} 94$

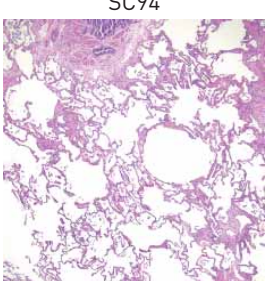

$\mathrm{SC} 93$

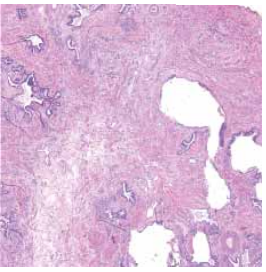

SC155

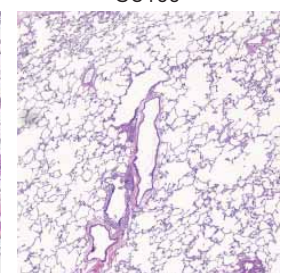

SC154

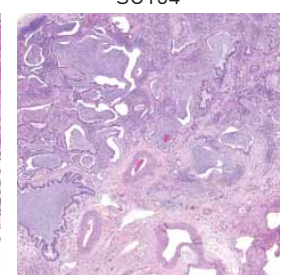

SC153

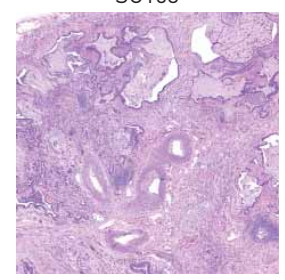

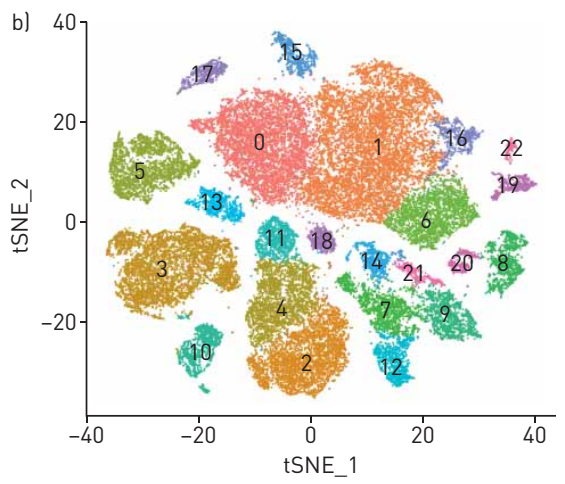

0 FABP $4^{\text {hi }}$ macrophage

1 SPP1 hi macrophage

2 T helper/T Reg
3 Endothelial

4 Cytotoxic T-cell

5 Fibroblast

6 FCN $^{\text {hi }}$ macrophage

7 AT1/club

8 Ciliated

9 Goblet

10 Smooth muscle/pericyte

11 NK cell

12 AT2

13 Mast cell

14 Basal

15 Proliferating cell

tSNE_1
16 Dendritic cell
17 Lymphatic endothelial
18 Mast cell
19 B-cell
20 Low quality ciliated
21 Low quality basal
22 Plasma cell
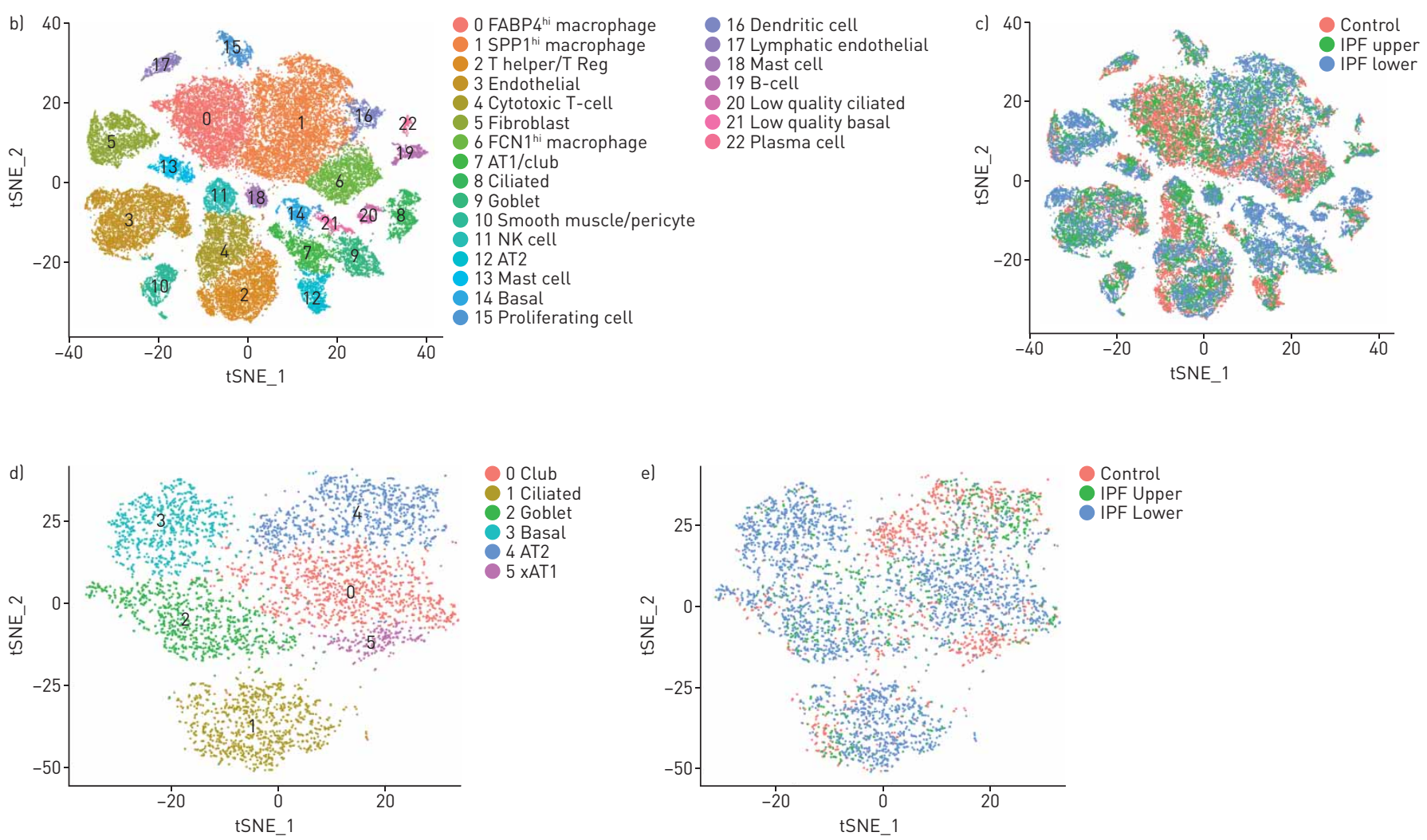

FIGURE 2 Combined t-distributed stochastic neighbour embedding (t-SNE) analysis of single-cell transcriptomes from three normal, three idiopathic pulmonary fibrosis (IPF) upper and three IPF lower lobes. a) Tissue sections of i) three control lung samples show preserved lung architecture with no significant pleural, subpleural or interstitial fibrosis and no increase in inflammatory cells. Tissue sections from patients with IPF showed iii) usual interstitial pneumonia lower lobes with a predominant cicatricial process and complete replacement of the lung architecture by scar tissue with honeycomb change, increased inflammatory cells and fibroblastic foci. ii) In comparison, the upper lobes showed relatively uninvolved lung tissue with preserved architecture and only mild interstitial organisation and mildly increased interstitial mononuclear cells suggestive of acute/subacute inflammation in samples SC88 and SC94 with more extensive fibrosis in SC154. Scale bar=1 mm. b) The t-SNE plot shows 20 clusters, with cell types identified by marker genes (supplementary figures S13 and S14). c,e) The tissue origin of the cells stratified by the type of tissue (control, IPF upper or IPF lower lobes) is indicated by different colouring of the cells. d) Reclustering of epithelial cells, showing the discrete clusters of club, ciliated (cluster 1) goblet and AT1 and AT2 cells, as identified by marker genes (supplementary figure S15). 
a)
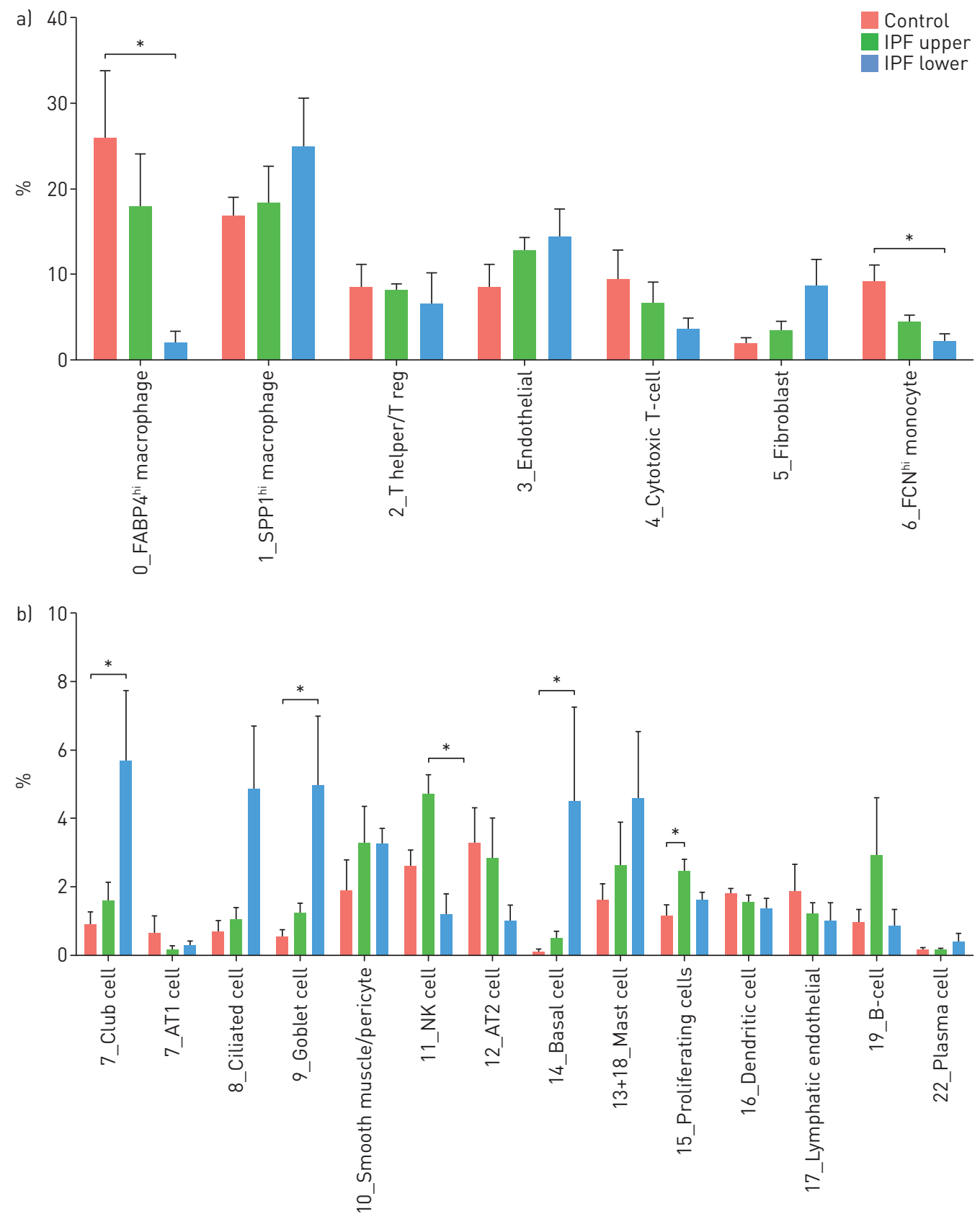

FIGURE 3 Percentages of cell types captured in control, upper idiopathic pulmonary fibrosis (IPF) and lower IPF lung samples. The average proportion of cell subgroups as a percentage of total cells analysed, in control, upper IPF lobes and lower IPF lobes are shown for each cluster as shown in figure $2 a$ and subclusters as shown in figure $2 \mathrm{~d} .{ }^{*}: \mathrm{p}<0.05$.

mesenchymal and alveolar epithelial cells compared to inflammatory cells (supplementary table S5). This skewing of cell types associated with cell survival during digestion was consistent between two control and two IPF samples analysed in this manner, indicating that although the absolute proportions of cells represented reflect the loss, the relative changes between samples are accurate.

Our results are generally consistent with a previous report (supplementary figure S19) in which epithelial cell adhesion molecule purified cells in normal lungs were mainly AT2 cells [21]. Ciliated, goblet, club and basal cells showed large increases in proportion of total cells in IPF. Fibroblasts, representing only $2 \%$ of cells in normal lungs, increased to $9 \%$ of cells in fibrotic lower lobes. Pericytes, endothelial cells and lymphatic endothelial cells showed little difference in cells numbers comparing IPF to control samples (figure $3 a$ and $b$ ). 

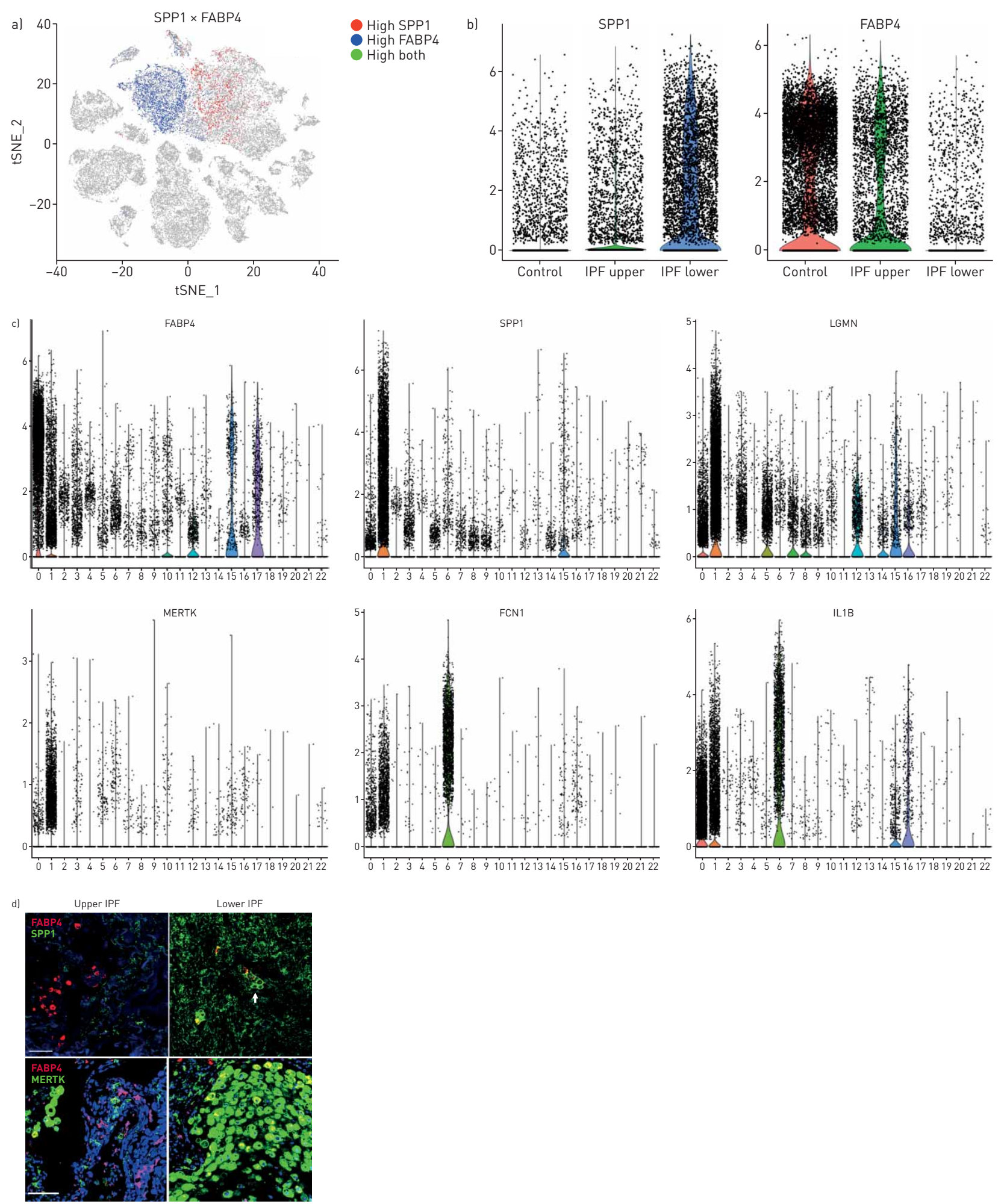

FIGURE 4 Macrophage populations in idiopathic pulmonary fibrosis (IPF) lungs. a) SPP1 and FABP4 expression define two discrete populations in IPF and normal lungs. b) land supplementary figure S18) SPP1 ${ }^{\text {hi }}$ macrophages express more SPP1 and make up a higher percentage of cells in lower lobes, while FABP4 ${ }^{\text {hi }}$ macrophages are a higher percentage of the cells in healthy and IPF upper lobes. c) Violin plots of combined IPF/ control lung data show expression of FABP4, SPP1, LGMN, MERTK, FCN1 and IL1B limited mainly to macrophage populations (clusters 0, FABP4 ${ }^{\text {hi }}$ macrophages; cluster 1 , SPP $1^{\text {hi }}$ macrophages and cluster 6, FCN1 ${ }^{\text {hi }}$ monocyte/macrophages; figure 2). d) (and supplementary figure S19) FABP4 expression is increased in FABP4 ${ }^{\text {hi }}$ macrophages, although it is also expressed at lower levels in SPP1 ${ }^{\text {hi }}$ macrophages; SPP1, MERTK and LGMN show highly increased expression in SPP1 ${ }^{\text {hi }}$ macrophages; FCN1 and IL1B are most highly expressed in FCN1 ${ }^{\text {hi }}$ monocyte/macrophages. Immunofluorescent staining for SPP1 shows macrophages embedded in the SPP1/osteopontin matrix; while staining for MERTK and FABP4 reveals these largely two discrete macrophage populations with increased FABP4-staining macrophages in upper lobes and increased MERTK macrophages in lower lobes. Scale bar $=100 \mu \mathrm{m}$. 
Macrophage subpopulations in IPF lungs

IPF upper lobes showed relatively few SPP $1^{\text {hi }}$ macrophages, and many FABP4 ${ }^{\text {hi }}$ macrophages, similar to control lungs (figure 4a and b). Macrophages expressing high levels of SPP1 and FABP4 were in large part mutually exclusive (figure $4 \mathrm{a}$ and $\mathrm{c}$ ). Immunofluorescent staining showed the same pattern of expression, with mainly FABP4-staining cells in the upper lobes, low level SPP1-staining and few MERTK-staining cells (figure $4 \mathrm{~d}$ and supplementary figure S21). IPF lower lobes showed higher numbers of SPP1-expressing cells compared to control or upper lobe IPF (figure 4a and $\mathrm{b}$ and supplementary figure S21). Immunofluorescent staining of IPF lower lobes showed much brighter diffuse staining for SPP1 as well as cells embedded in the SPP1 matrix, many MERTK-staining cells and relatively few FABP4-staining cells (figure 4c, supplementary figure S21). Staining of serial sections of lower lobes confirmed that the three macrophage populations were distinct and co-stained with CD163 (supplementary figure S22).

SPP1 stained much more highly in IPF lower lobe than upper lobe, staining most intensely surrounding and within fibroblastic foci (figure 5a, supplementary figure S23). MERTK discretely stained macrophages surrounding and within fibroblastic foci.

\section{Gene regulation in IPF fibroblasts}

Altered average gene expression was associated with each cluster in IPF upper and lower lobes and healthy lungs (table S6; https://dom.pitt.edu/rheum/centers-institutes/scleroderma/systemicsclerosiscenter/database/). IPF fibroblasts showed many highly upregulated matrix genes, including TNN (no denominator, nd), COL10A1 (612-fold), COMP (17-fold), POSTN (470-fold), COL1A1 (170-fold). Wnt-related genes that are expressed by dermal fibroblast subtypes [22] were also upregulated, SFRP4 (5.1-fold) and SFRP2 (8.8-fold) (figure 5b).

\section{Genes upregulated in SPP1 macrophages in IPF lungs}

To highlight genes regulated by SPP1 macrophages in IPF patients, we ranked genes by fold change between IPF and normal SPP $1{ }^{\text {hi }}$ macrophages. To limit detection of ambient RNA, we selected genes that were more highly expressed by lower lobe IPF SPP $1{ }^{\text {hi }}$ macrophages than any other cell type, and low-level expressed genes (expressed at an average level of $\leqslant 0.01$ by SPP $1^{\text {hi }}$ lower lobe macrophages) were excluded (supplementary table S7). The most highly upregulated genes in lower lobe IPF compared to control lungs included LEP (nd), KCNJ5 (15.76-fold), HS3ST2 (15.58-fold), SPP1 (7.56-fold), SIGLEC15 (7.43-fold), ATP6VOD2 (7.12-fold), LGMN (6.07-fold), MERTK (4.96-fold) and MMP9 (3.81-fold). LEP, HS3ST2, SPP1, SIGLEC15, LGMN, MERTK, LGMN and MMP9 were most specifically upregulated in IPF SPP $1^{\text {hi }}$ and proliferating macrophages (supplementary figure S24). Many other genes that were highly upregulated in SPP $1{ }^{\text {hi }}$ IPF macrophages showed a graded increase in gene expression comparing control macrophages to upper lobe IPF macrophages to lower lobe IPF macrophages, suggesting a continuum in differentiation of these cells throughout disease progression (supplementary table S8)

We examined IL4, IL13, MRC1 and TMG2 expression, the latter two genes markers of human M2 macrophages $[23,24]$. Although expressed at very low levels, IL4 was expressed and upregulated mainly T-cells in IPF compared to controls, whereas IL13 expression was downregulated in mast cells (supplementary figure S25). TGM2 was modestly upregulated in all IPF macrophage subsets, although much more highly expressed in non-macrophage cell types. MRC1 was modestly downregulated in all macrophage cell types.

\section{Proliferating cells in IPF lungs}

We found in cluster 15 from control/IPF lung clustering (figure 2b) cells expressing highest levels of G2/M markers and specific markers of cell proliferation, including MKI67 (i.e. Ki67), KIAA0101/PCLAF (PCNA-associated factor), BIRC5 (survivin) and UBE2C/UBCH10 ubiquitin-conjugating enzyme E2 C (figure $6 \mathrm{~b}$ and $\mathrm{d}$ ). Both FAPB $4^{\mathrm{hi}}$ and $\mathrm{SPP} 1^{\text {hi }}$ macrophages were proliferating. More FAPB4 ${ }^{\text {hi }}$ macrophages were proliferating in the IPF upper lobe, but when adjusted for the paucity of these cells in lower lobes, the percentage of proliferating cells was similar (10.59\% compared to $16.95 \%$, supplementary table S4). SPP $1{ }^{\text {hi }}$ macrophages were rarely proliferating in healthy lungs $(0.072 \%)$, but showed dramatically increased proliferation in the IPF upper $\left(2.01 \%\right.$ of SPP $1^{\text {hi }}$ macrophages) and lower lobes $\left(3.50 \%\right.$ of SPP $1^{\text {hi }}$ macrophages; figure $6 \mathrm{c}$ and e and supplementary table S4), representing the most common proliferating macrophage in IPF lower lobes $(2.8 \%$ compared to $1.7 \%$ and $0.3 \%$ proliferating FABP4 and FCN1, respectively, of total macrophages, table S4). Epithelial cells were also proliferating. Low numbers of proliferating AT1, AT2, club and ciliated cells did not clearly trend toward altered rates of proliferation in IPF (table S4, figures S26 and S27). However, KRT5 ${ }^{+}$basal cells showed a clear trend toward increased proliferation with control, IPF upper and IPF lower lungs showing, respectively, none (0\%), one $(1.49 \%)$ and 33 (4.64\%) of basal cells proliferating. These cells also showed highly upregulated expression of TP63 ( $\mathrm{p} 63$, supplementary figure S28). 

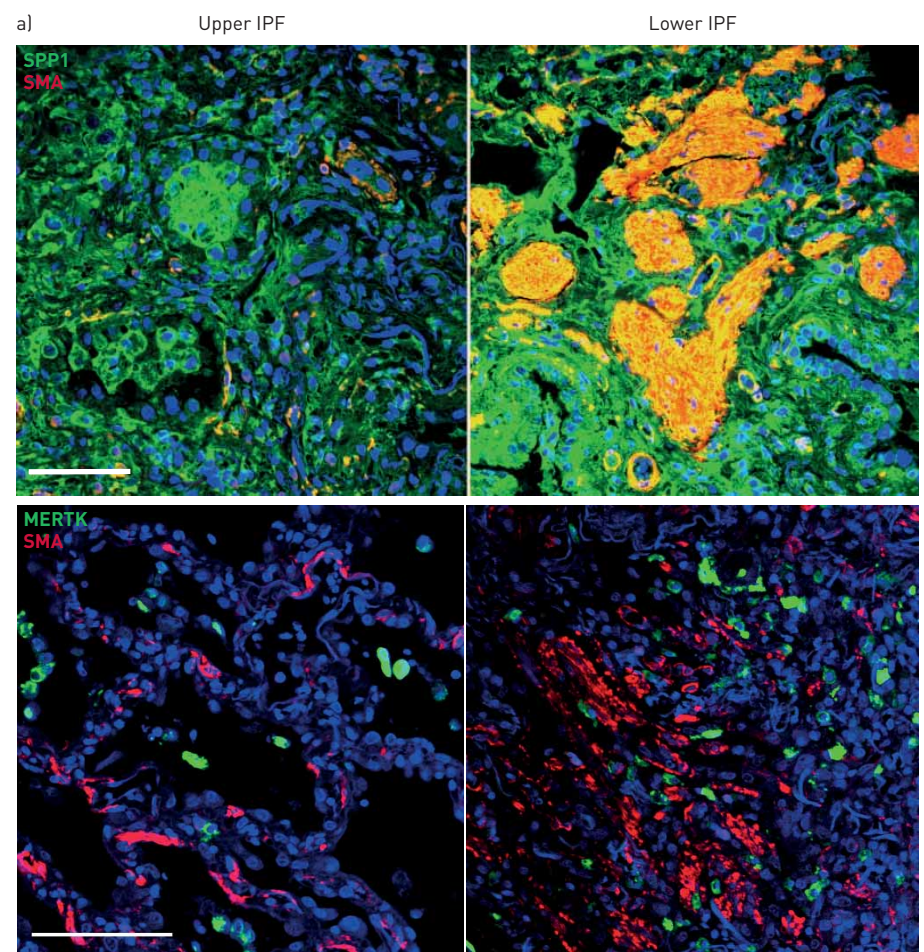

COL1A1

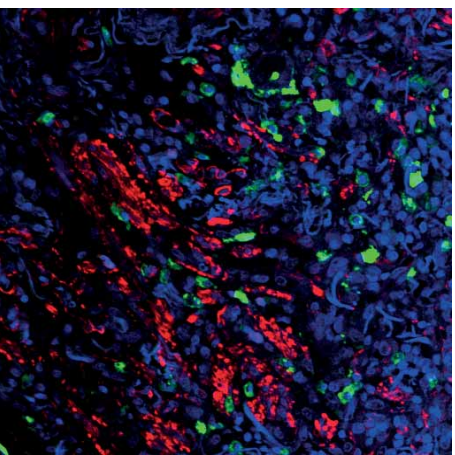

b)
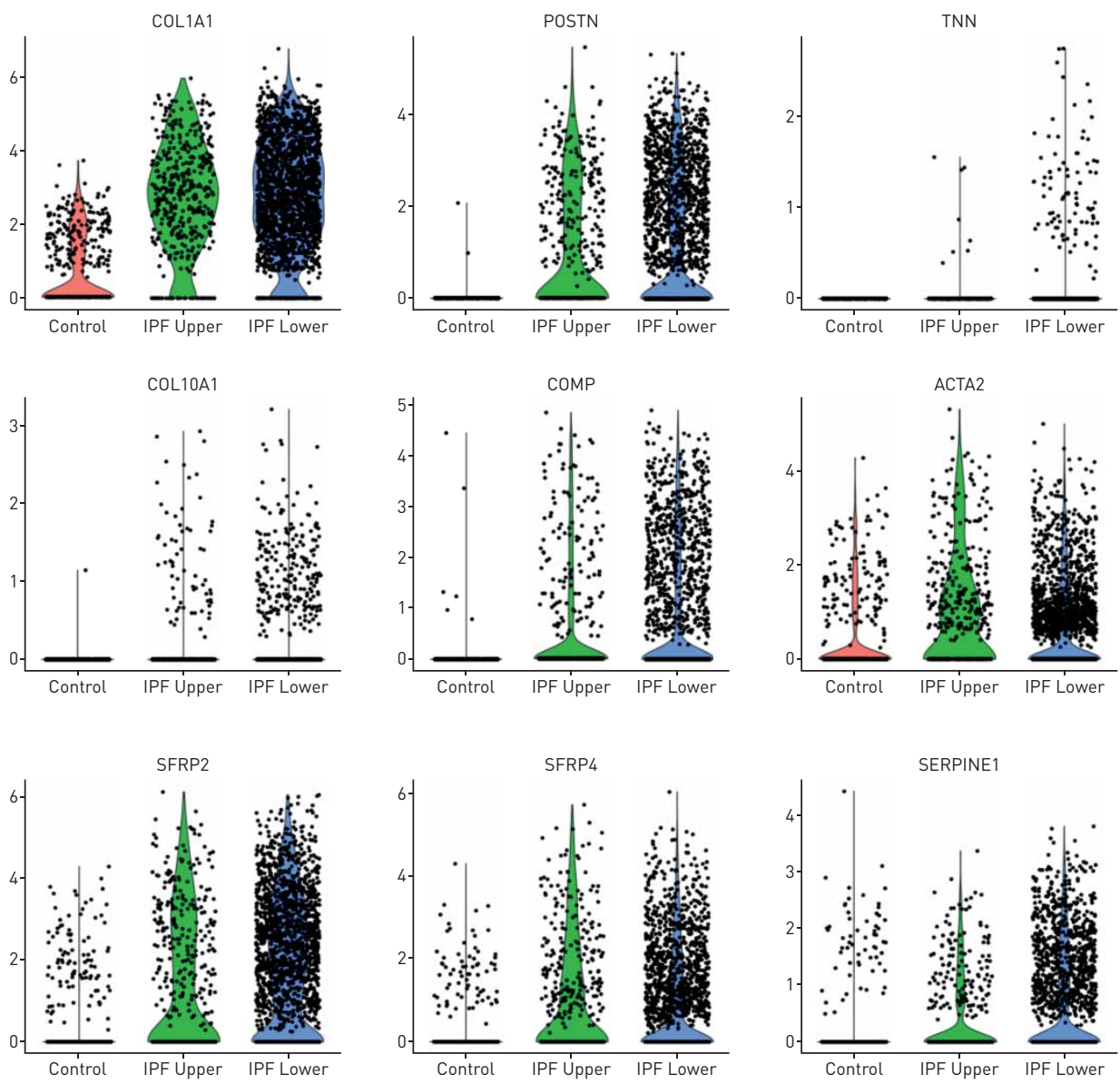

FIGURE 5 SPP1 macrophages and myofibroblasts in idiopathic pulmonary fibrosis (IPF) lungs. a) Fibroblastic foci in IPF lungs were stained with $\alpha$-smooth muscle actin (SMA; red). Macrophages were stained with SPP1 or MERTK as indicated (green). b) Violin plots, indicating expression of several genes associated with fibrosis in control, IPF upper and IPF lower lungs. 

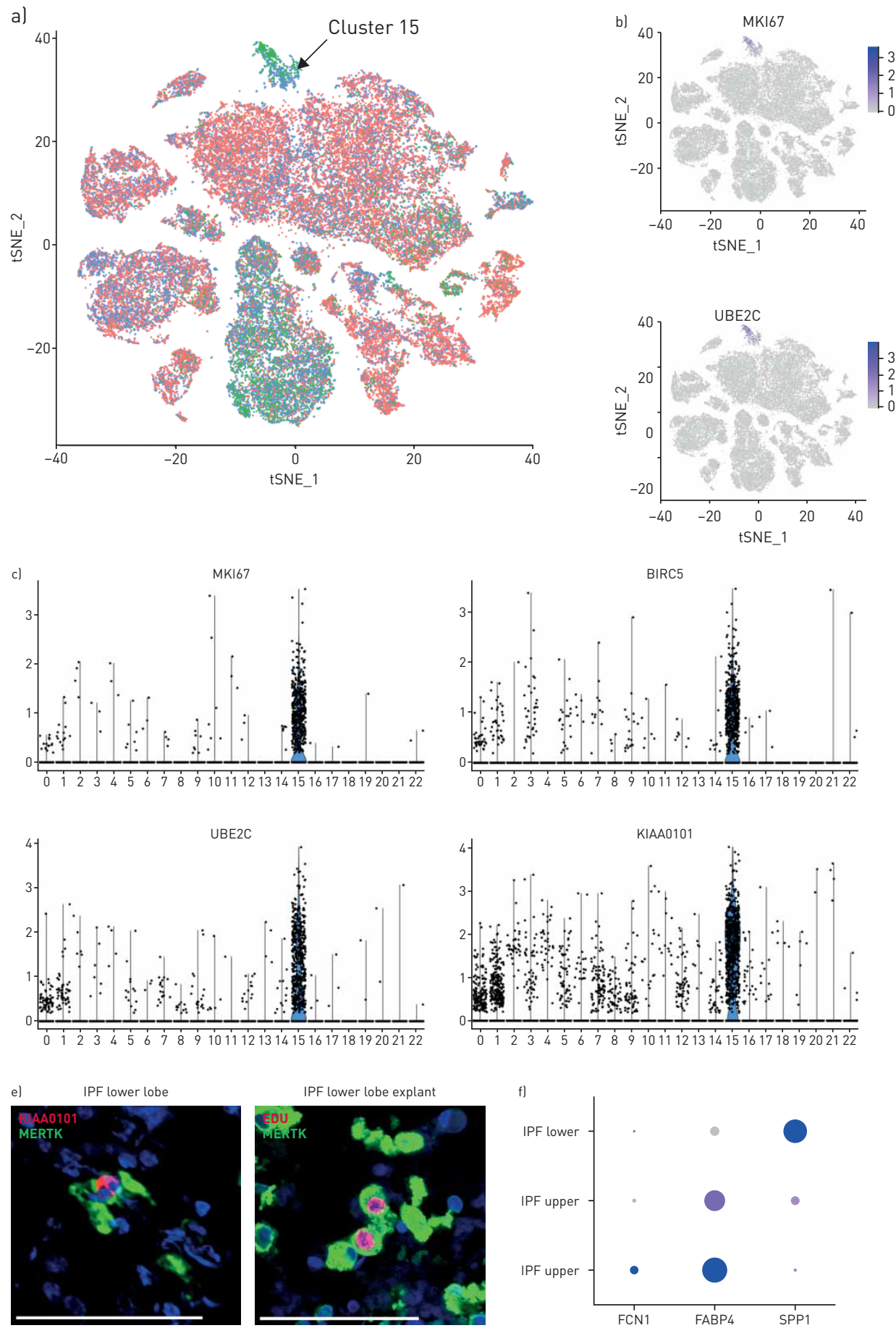

FIGURE 6 Proliferating cells in idiopathic pulmonary fibrosis (IPF) and normal lungs. a) Proliferating cells from control and IPF lungs form a distinct cluster (cluster 15, green G2/M phase cells marked by arrow; see also figure 2b, cluster 15). b, c) Cluster 15 cells highly express MKI67 (Ki-67), BIRC5, UBE2C and KIAA0101. d) Reclustering of cluster 15 cells shows macrophages expressing either FABP4 or SPP1. Using KIAA as a marker for proliferating cells, both FABP4 and SPP1 ${ }^{\text {hi }}$ cells are proliferating in this cluster. e) MERTK-expressing macrophages from IPF lower lobe lung explant co-stained with MERTK and proliferation marker KIAA0101. Macrophages labelled in vitro by incubation with EdU (red), co-stained by immunofluorescent with MERTK (green). f) Examining gene expression by only the proliferating macrophage subset shows low level proliferation of FCN1/(IL1B-expressing) macrophages in control lungs; primarily proliferation of FCN1/(IL1B-expressing) macrophages and FABP $4^{\text {hi }}$ macrophages in IPF upper lobes and primarily proliferation of SPP1 ${ }^{\text {hi }}$ macrophages in IPF lower lobes (size of dot indicates the proportion of cells and intensity of purple the relative level of gene expression). Scale bar $=100 \mu \mathrm{m}$.

Proliferating cells were identified by KIAA0101/PCLAF staining by immunofluorescence. KIAA0101/ PCLAF cells co-expressed SPP1 in lower lobes and FABP4 in the upper lobes (figure 6e, supplementary figure S14B). Proliferating IPF cells in explant culture, incorporating EdU (5-ethynyl-2'-deoxyuridine), 
co-stained with CD163, confirming proliferating macrophages (figure 6e). Expression of CSF1, whose gene product CSF-1 is known to stimulate monocyte/macrophage proliferation, was strongly upregulated in IPF mast cells (supplementary figure S25).

\section{Connectivity maps of cell subtypes}

We built a graphical model to observe the direct relationships between differentially expressed genes for SPP1/MERTK macrophages, fibroblasts and various epithelial cell types. The resulting network showed the most densely connected gene groups were between SPP1 macrophages and fibroblasts, suggesting a causal relationship between these two cell types (supplementary figure S29, table S9 and supplementary results).

In addition, we examined gene expression associated with each of the macrophage populations using gene ontology (GO) analysis. FABP4 ${ }^{\text {hi }}$ macrophages expressed genes involved in lipid metabolism more highly, $\mathrm{SPP}^{\mathrm{hi}}$ macrophages expressed genes involved in the stress response more highly and $\mathrm{FCN1}{ }^{\mathrm{hi}}$ macrophages expressed genes associated with the immune response more highly (supplementary table S10). In addition, we compared GO terms activated in IPF compared to control IPF macrophages, showing upregulated expression of genes associated with extracellular matrix organisation.

\section{Discussion}

SPP1/osteopontin is a consistently observed marker of IPF [25]. Our results show that SPP1 is a highly selective marker for an expanded subpopulation of macrophages found in human IPF. SPP1 is selectively expressed by a subgroup of macrophages found in normal healthy lungs but seen rarely in BAL, indicating that they are part of the interstitial macrophage compartment. In contrast to another recent description of scRNA-seq data in IPF [26], our data indicate that SPP $1{ }^{\text {hi }}$ macrophages do not arise as a new macrophage population in IPF, but are present in normal lungs as well. In our reclustering of the data from this previous study [26], we found similar populations of SPP1 $1^{\text {hi }}, \mathrm{FABP}_{4}{ }^{\mathrm{hi}}$ and $\mathrm{FCN1}{ }^{\text {hi }}$ macrophages in normal lungs, indicating that $\mathrm{SPP} 1^{\mathrm{hi}}$ cells in IPF probably arise from a macrophage population already present in normal lungs. However, SPP1 expression increased dramatically in this macrophage subset in IPF, particularly in the fibrotic IPF lower lobe. The SPP1 gene product, osteopontin, was strikingly deposited in fibrotic IPF lower lobes, where it was associated with fibroblastic foci. Osteopontin supports monocyte/ macrophage proliferation [27], suggesting that macrophage secretion of this matrix protein might support SPP1 macrophage proliferation in IPF. Deletion of SPP1 in bleomycin-induced lung fibrosis reduces upregulated expression of collagen type 1 and MMP2 [28, 29]. SPP1 deletion ameliorates bleomycin-induced dermal fibrosis and carbon nanotube-induced lung fibrosis [30, 31]. Osteopontin is increased in the serum of patients with systemic sclerosis [30], and has been implicated in renal cardiac and bone marrow fibrosis, suggesting that SPP1 macrophages may have a more general role in promoting fibrosis [32-35]. We propose that SPP $1^{\text {hi }}$ macrophages represent a profibrotic macrophage population in IPF lungs.

Highly increased co-expression of SPP1 and MERTK by SPP $1^{\text {hi }}$ macrophages may be key to a role for these cells in IPF tissue repair and fibrosis. MERTK is a receptor for complexes of Gas6 or protein $S$ bound to phosphatidylserine, exposed on apoptotic cells [36, 37], and the main apoptotic cell receptor on macrophages [38, 39]. Alveolar cell apoptosis is a feature of IPF [40], and blocking Gas6 inhibits markers of fibroblast activation [41]. Macrophage efferocytosis suppresses inflammation, in part through upregulated transforming growth factor- $\beta$, prostaglandin E2 and platelet-activating factor [42]. In addition, MERTK engagement by apoptotic cells stimulates macrophage production of pro-resolving lipid mediators [43]. Supporting the notion that $S P P 1^{\text {hi }}$ macrophages are reparative macrophages, MERTK macrophages aid tissue repair after cardiac or liver injury [44, 45]. As inhibiting MERTK leads to apoptosis of myeloid cells [46], MERTK inhibitors under clinical development [46-48] might deplete profibrotic macrophage and fibroblast activation in IPF lungs. In the central nervous system microglial cells not only clear apoptotic cells, but also target live, damaged cells [49], referred to as phagoptosis [50, 51]. Thus, macrophages expressing increased MERTK might phagocytose live, damaged or senescent alveolar epithelial cells.

FABP4 ${ }^{\text {hi }}$ macrophages represented a second subset of lung macrophages, making up most of the macrophages in alveoli showed dramatically decreased numbers in IPF in lower lobes. FABP4 expression by macrophages induces inflammation associated with obesity and atherosclerosis [52, 53]. Deletion of macrophage FABP4 is associated with increased intracellular fatty acid and increased unfolded protein response [54]. In other studies, FABP4 expression in macrophages is associated with pro-inflammatory macrophages and IL1 $\beta$ secretion [55].

FCN1 $^{\text {hi }}$ macrophages represented a third population of macrophages with marker closely related to monocytes. Although these could include true intravascular monocytes, they were not consistently seen in blood vessels in IHC; the normal healthy lungs were always on ex vivo lung perfusion prior to harvest, 
probably washing out most or all blood associated monocytes; and a small fraction were captured in BAL. Thus, they probably represent a third macrophage subpopulation found primarily in the interstitial compartment. Complementary expression of several marker genes suggests that SPP1 $1^{\text {hi }}, \mathrm{FABP} 4^{\text {hi }}$ and FCN1 ${ }^{\text {hi }}$ macrophages represent discrete macrophage subsets.

Both FAPB $4^{\text {hi }}$ and SPP $1^{\text {hi }}$ macrophage subsets increased their rate of proliferation in IPF lungs, SPP $1^{\text {hi }}$ macrophages particularly in fibrotic lower lobes. $\mathrm{FCN1}^{\text {hi }}$ monocyte/macrophages showed lower rates of proliferation that were relatively constant between healthy and IPF lungs $(\sim 2 \%)$. Observed basal proliferation of both $\mathrm{FCN}_{1}{ }^{\mathrm{hi}}$ and $\mathrm{FABP} 4^{\mathrm{hi}}$ macrophages supports some degree of self-renewal of these populations in healthy lungs.

Tissue resident macrophages self-renew under the influence of M-CSF and/or GM-CSF [15, 16, 18, 56]. We found increased expression of CSF1 in lung mast cells, suggesting that this might contribute to increased macrophage proliferation in IPF. In addition, IL-4 stimulates resident macrophage proliferation, suggesting that proliferation might be a feature of M2 macrophages [17]. IL-4/IL-13 have been implicated in murine lung fibrosis [57, 58]. We saw increased IL4 mRNA expression by IPF T-cells, although low-level expression of IL4 and IL13 make these results uncertain. In IPF lungs, SPP $1^{\text {hi }}$ macrophages upregulated DC-SIGN (CD209), LGMN and CHI3L1, all regulated by IL-4 and/or IL-13 [59-62]. Together these results are consistent with possible CSF-1-, IL-4- and/or IL-13-induced local macrophage proliferation.

Despite increased proliferation of $\mathrm{FABP} 4^{\mathrm{hi}}$ macrophages, the total percentage of these cells decreased particularly in IPF lower lobes. Thus, proliferating FAPB4 ${ }^{\text {hi }}$ macrophages appear to be either dying or changing phenotype. We speculate that FAPB $4^{\text {hi }}$ macrophages, which are the primary cell type in alveoli, might die in fibrotic IPF lower lobes. Alternatively, these cells might transition into SPP $1^{\text {hi }}$ macrophages in IPF lungs. In summary, our data show striking changes in cell populations in IPF that include marked increases in local proliferation of two populations of macrophages, one of which is highly associated with IPF diseased lungs.

Acknowledgements: The authors acknowledge the assistance of William Horne (Children's Hospital of Pittsburgh, Pittsburgh, PA, USA) for helpful discussions and technical support for RNA-sequencing.

Author contributions: C. Morse performed immunofluorescence; T. Tabib performed single-cell RNA-sequencing experiments and bioinformatics analyses; J. Sembrat procured human tissues; K. Buschur analysed data by graphical modelling; H. Trejo Bittar reviewed and confirmed lung pathology; Y. Jiang helped with bioinformatics analyses; D.J. Kass provided intellectual support; E. Valenzi and K. Gibson reviewed clinical data; P.V. Benos supervised graphical modelling; W. Chen supervised RNA-sequencing analysis; C. Morse, T. Tabib, A. Mora, M. Rojas and R. Lafyatis conceived and guided the study; R. Lafyatis wrote the manuscript. All authors reviewed, edited and approved the final manuscript.

Support statement: Research reported in this publication was supported by the National Institute of Arthritis and Musculoskeletal and Skin Diseases under award number 2P50 AR060780; National Institute for Heart, Lung, and Blood Institute under award number R01 HL123766, U01 HL137159, R01 HL131789 and U0 HL137159; and National Library of Medicine under award R01 LM012087 of the National Institutes of Health. The content is solely the responsibility of the authors and does not necessarily represent the official views of the National Institutes of Health. Funding information for this article has been deposited with the Crossref Funder Registry.

Conflict of interest: C. Morse has nothing to disclose. T. Tabib has nothing to disclose. J. Sembrat has nothing to disclose. K. Buschur has nothing to disclose. H. Trejo Bittar has nothing to disclose. E. Valenzi has nothing to disclose. Y. Jiang has nothing to disclose. D.J. Kass reports grants from NIH, during the conduct of the study; grants from Regeneron, outside the submitted work. K. Gibson has nothing to disclose. W. Chen has nothing to disclose. A. Mora has nothing to disclose. P.V. Benos has nothing to disclose. M. Rojas has nothing to disclose. R. Lafyatis has nothing to disclose.

\section{References}

1 Blackwell TS, Tager AM, Borok Z, et al. Future directions in idiopathic pulmonary fibrosis research. An NHLBI workshop report. Am J Respir Crit Care Med 2014; 189: 214-222.

2 Sisson TH, Mendez M, Choi K, et al. Targeted injury of type II alveolar epithelial cells induces pulmonary fibrosis. Am J Respir Crit Care Med 2010; 181: 254-263.

3 Zoz DF, Lawson WE, Blackwell TS. Idiopathic pulmonary fibrosis: a disorder of epithelial cell dysfunction. Am J Med Sci 2011; 341: 435-438.

4 Evans CM, Fingerlin TE, Schwarz MI, et al. Idiopathic pulmonary fibrosis: a genetic disease that involves mucociliary dysfunction of the peripheral airways. Physiol Rev 2016; 96: 1567-1591.

5 Rock JR, Gao X, Xue Y, et al. Notch-dependent differentiation of adult airway basal stem cells. Cell Stem Cell 2011; 8: 639-648.

6 Rock JR, Onaitis MW, Rawlins EL, et al. Basal cells as stem cells of the mouse trachea and human airway epithelium. Proc Natl Acad Sci USA 2009; 106: 12771-12775.

7 Vaughan AE, Brumwell AN, Xi Y, et al. Lineage-negative progenitors mobilize to regenerate lung epithelium after major injury. Nature 2015; 517: 621-625. 
8 Kulkarni T, O'Reilly P, Antony VB, et al. Matrix remodeling in pulmonary fibrosis and emphysema. Am J Respir Cell Mol Biol 2016; 54: 751-760.

9 Okuma T, Terasaki Y, Kaikita K, et al. C-C chemokine receptor 2 (CCR2) deficiency improves bleomycin-induced pulmonary fibrosis by attenuation of both macrophage infiltration and production of macrophage-derived matrix metalloproteinases. J Pathol 2004; 204: 594-604.

10 McCubbrey AL, Barthel L, Mohning MP, et al. Deletion of c-FLIP from CD11b $b^{\text {hi }}$ macrophages prevents development of bleomycin-induced lung fibrosis. Am J Respir Cell Mol Biol 2017; 58: 66-78.

11 Misharin AV, Morales-Nebreda L, Reyfman PA, et al. Monocyte-derived alveolar macrophages drive lung fibrosis and persist in the lung over the life span. J Exp Med 2017; 214: 2387-2404.

12 Guilliams M, De Kleer I, Henri S, et al. Alveolar macrophages develop from fetal monocytes that differentiate into long-lived cells in the first week of life via GM-CSF. J Exp Med 2013; 210: 1977-1992.

13 Schulz C, Gomez Perdiguero E, Chorro L, et al. A lineage of myeloid cells independent of Myb and hematopoietic stem cells. Science 2012; 336: 86-90.

14 Jakubzick C, Gautier EL, Gibbings SL, et al. Minimal differentiation of classical monocytes as they survey steady-state tissues and transport antigen to lymph nodes. Immunity 2013; 39: 599-610.

15 Hashimoto D, Chow A, Noizat C, et al. Tissue-resident macrophages self-maintain locally throughout adult life with minimal contribution from circulating monocytes. Immunity 2013; 38: 792-804.

16 Davies LC, Rosas M, Smith PJ, et al. A quantifiable proliferative burst of tissue macrophages restores homeostatic macrophage populations after acute inflammation. Eur J Immunol 2011; 41: 2155-2164.

17 Jenkins SJ, Ruckerl D, Cook PC, et al. Local macrophage proliferation, rather than recruitment from the blood, is a signature of TH2 inflammation. Science 2011; 332: 1284-1288.

18 Davies LC, Jenkins SJ, Allen JE, et al. Tissue-resident macrophages. Nat Immunol 2013; 14: 986-995.

19 Seibold MA, Smith RW, Urbanek C, et al. The idiopathic pulmonary fibrosis honeycomb cyst contains a mucocilary pseudostratified epithelium. PLoS One 2013; 8: e58658.

20 Treutlein B, Brownfield DG, Wu AR, et al. Reconstructing lineage hierarchies of the distal lung epithelium using single-cell RNA-seq. Nature 2014; 509: 371-375.

$21 \mathrm{Xu}$ Y, Mizuno T, Sridharan A, et al. Single-cell RNA sequencing identifies diverse roles of epithelial cells in idiopathic pulmonary fibrosis. JCI insight 2016; 1: e90558.

22 Tabib T, Morse C, Wang T, et al. SFRP2/DPP4 and FMO1/LSP1 define major fibroblast populations in human skin. J Invest Dermatol 2018; 138: 802-810.

23 Christmann RB, Hayes E, Pendergrass S, et al. Interferon and alternative activation of monocyte/macrophages in systemic sclerosis-associated pulmonary arterial hypertension. Arthritis Rheum 2011; 63: 1718-1728.

24 Martinez FO, Helming L, Milde R, et al. Genetic programs expressed in resting and IL-4 alternatively activated mouse and human macrophages: similarities and differences. Blood 2013; 121: e57-e69.

25 Pardo A, Gibson K, Cisneros J, et al. Up-regulation and profibrotic role of osteopontin in human idiopathic pulmonary fibrosis. PLoS Med 2005; 2: e251.

26 Reyfman PA, Walter JM, Joshi N, et al. Single-cell transcriptomic analysis of human lung provides insights into the pathobiology of pulmonary fibrosis. Am J Respir Crit Care Med 2018; 12: 1517-1536.

27 Tardelli M, Zeyda K, Moreno-Viedma V, et al. Osteopontin is a key player for local adipose tissue macrophage proliferation in obesity. Mol Metab 2016; 5: 1131-1137.

28 Berman JS, Serlin D, Li X, et al. Altered bleomycin-induced lung fibrosis in osteopontin-deficient mice. Am Physiol Lung Cell Mol Physiol 2004; 286: L1311-L1318.

29 Takahashi F, Takahashi K, Okazaki T, et al. Role of osteopontin in the pathogenesis of bleomycin-induced pulmonary fibrosis. Am J Respir Cell Mol Biol 2001; 24: 264-271.

30 Wu M, Schneider DJ, Mayes MD, et al. Osteopontin in systemic sclerosis and its role in dermal fibrosis. J Invest Dermatol 2012; 132: 1605-1614.

31 Dong J, Ma Q. Osteopontin enhances multi-walled carbon nanotube-triggered lung fibrosis by promoting TGF- $\beta 1$ activation and myofibroblast differentiation. Part Fibre Toxicol 2017; 14: 18.

32 Collins AR, Schnee J, Wang W, et al. Osteopontin modulates angiotensin II-induced fibrosis in the intact murine heart. J Am Coll Cardiol 2004; 43: 1698-1705.

33 Merszei J, Wu J, Torres L, et al. Osteopontin overproduction is associated with progression of glomerular fibrosis in a rat model of anti-glomerular basement membrane glomerulonephritis. Am J Nephrol 2010; 32: 262-271.

34 Ruberti S, Bianchi E, Guglielmelli P, et al. Involvement of MAF/SPP1 axis in the development of bone marrow fibrosis in PMF patients. Leukemia 2018; 32: 438-449.

35 Zahradka P. Novel role for osteopontin in cardiac fibrosis. Circ Res 2008; 102: 270-272.

36 Scott RS, McMahon EJ, Pop SM, et al. Phagocytosis and clearance of apoptotic cells is mediated by MER. Nature 2001; 411: 207-211.

37 van der Meer JH, van der Poll T, van 't Veer C. TAM receptors, Gas6, and protein S: roles in inflammation and hemostasis. Blood 2014; 123: 2460-2469.

38 Sather S, Kenyon KD, Lefkowitz JB, et al. A soluble form of the Mer receptor tyrosine kinase inhibits macrophage clearance of apoptotic cells and platelet aggregation. Blood 2007; 109: 1026-1033.

39 Seitz HM, Camenisch TD, Lemke G, et al. Macrophages and dendritic cells use different Axl/Mertk/Tyro3 receptors in clearance of apoptotic cells. J Immunol 2007; 178: 5635-5642.

40 Thannickal VJ, Horowitz JC. Evolving concepts of apoptosis in idiopathic pulmonary fibrosis. Proc Am Thorac Soc 2006; 3: 350-356.

41 Espindola MS, Habiel DM, Narayanan R, et al. Targeting of TAM receptors ameliorates fibrotic mechanisms in idiopathic pulmonary fibrosis. Am J Respir Crit Care Med 2018; 197: 1443-1456.

42 Fadok VA, Bratton DL, Konowal A, et al. Macrophages that have ingested apoptotic cells in vitro inhibit proinflammatory cytokine production through autocrine/paracrine mechanisms involving TGF-beta, PGE2, and PAF. J Clin Invest 1998; 101: 890-898.

43 Cai B, Thorp EB, Doran AC, et al. MerTK cleavage limits proresolving mediator biosynthesis and exacerbates tissue inflammation. Proc Natl Acad Sci USA 2016; 113: 6526-6531.

44 DeBerge M, Yeap XY, Dehn S, et al. MerTK cleavage on resident cardiac macrophages compromises repair after myocardial ischemia reperfusion injury. Circ Res 2017; 121: 930-940. 
Triantafyllou E, Pop OT, Possamai LA, et al. MerTK expressing hepatic macrophages promote the resolution of inflammation in acute liver failure. Gut 2018; 67: 333-347.

Koda Y, Itoh M, Tohda S. Effects of MERTK inhibitors UNC569 and UNC1062 on the growth of acute myeloid leukaemia cells. Anticancer Res 2018; 38: 199-204.

47 Wu J, Frady LN, Bash RE, et al. MerTK as a therapeutic target in glioblastoma. Neuro Oncol 2018; 20: 92-102.

48 Yi JH, Jang J, Cho J, et al. MerTK is a novel therapeutic target in gastric cancer. Oncotarget 2017; 8: 96656-96667.

49 Fourgeaud L, Través PG, Tufail Y, et al. TAM receptors regulate multiple features of microglial physiology. Nature 2016; 532: 240-244.

50 Brown GC, Neher JJ. Eaten alive! Cell death by primary phagocytosis: 'phagoptosis'. Trends Biochem Sci 2012; 37: 325-332.

51 Brown GC, Neher JJ. Microglial phagocytosis of live neurons. Nat Rev Neurosci 2014; 15: $209-216$.

52 Makowski L, Boord JB, Maeda K, et al. Lack of macrophage fatty-acid-binding protein aP2 protects mice deficient in apolipoprotein E against atherosclerosis. Nat Med 2001; 7: 699-705.

53 Hotamisligil GS, Bernlohr DA. Metabolic functions of FABPs - mechanisms and therapeutic implications. Nat Rev Endocrinol 2015; 11: 592-605.

$54 \mathrm{Xu} \mathrm{H}$, Hertzel AV, Steen KA, et al. Uncoupling lipid metabolism from inflammation through fatty acid binding protein-dependent expression of UCP2. Mol Cell Biol 2015; 35: 1055-1065.

55 Steen $\mathrm{KA}, \mathrm{Xu} \mathrm{H}$, Bernlohr DA. FABP4/aP2 regulates macrophage redox signaling and inflammasome activation via control of UCP2. Mol Cell Biol 2017; 37: e00282-16.

56 Antonov AS, Munn DH, Kolodgie FD, et al. Aortic endothelial cells regulate proliferation of human monocytes in vitro via a mechanism synergistic with macrophage colony-stimulating factor. Convergence at the cyclin E/p27 (Kip1) regulatory checkpoint. J Clin Invest 1997; 99: 2867-2876.

57 Huaux F, Liu T, McGarry B, et al. Dual roles of IL-4 in lung injury and fibrosis. J Immunol 2003; 170 : $2083-2092$.

58 Lee CG, Homer RJ, Zhu Z, et al. Interleukin-13 induces tissue fibrosis by selectively stimulating and activating transforming growth factor $\beta_{1}$. J Exp Med 2001; 194: 809-821.

59 Relloso M, Puig-Kröger A, Pello OM, et al. DC-SIGN (CD209) expression is IL-4 dependent and is negatively regulated by IFN, TGF- $\beta$, and anti-inflammatory agents. J Immunol 2002; 168: 2634-2643.

60 Soilleux EJ, Morris LS, Leslie G, et al. Constitutive and induced expression of DC-SIGN on dendritic cell and macrophage subpopulations in situ and in vitro. J Leukoc Biol 2002; 71: 445-457.

61 Lee CG, Hartl D, Lee GR, et al. Role of breast regression protein 39 (BRP-39)/chitinase 3-like-1 in Th2 and IL-13-induced tissue responses and apoptosis. J Exp Med 2009; 206: 1149-1166.

62 Sakazaki Y, Hoshino T, Takei S, et al. Overexpression of chitinase 3-like 1/YKL-40 in lung-specific IL-18-transgenic mice, smokers and COPD. PLoS One 2011; 6: e24177. 